\title{
Antioxidant capacity of lipid- and water- soluble antioxidants in dogs with subclinical myxomatous mitral valve degeneration anaesthetised with propofol or sevoflurane
}

Katerina Tomsič ${ }^{1 \dagger}$, Alenka Nemec Svete ${ }^{1 \dagger}$, Ana Nemec ${ }^{1}$, Aleksandra Domanjko Petrič ${ }^{1}$, Tatjana Pirman², Vida Rezar², Tomaž Vovk ${ }^{3}$ and Alenka Seliškar ${ }^{1 *}$ (i)

\begin{abstract}
Background: Antioxidants located in both the hydrophilic and lipophilic compartments of plasma act as a defence system against reactive oxygen species (ROS). Excessive production of ROS during anaesthesia affects the antioxidant capacity of plasma and may result in oxidative stress. The aim of this study was to evaluate the antioxidant capacity of lipid- (ACL) and water-soluble (ACW) antioxidants in client-owned dogs diagnosed with periodontal disease and early-stage myxomatous mitral valve degeneration (MMVD) and anaesthetised for a dental procedure with propofol and sevoflurane or with propofol only.

Results: Dogs with MMVD were anaesthetised with propofol and sevoflurane (MMVD/PS, $n=8$ ) or with propofol only (MMVD/P, $n=10)$. Dogs with no evidence of MMVD (PS, $n=12)$ were anaesthetised with propofol and sevoflurane. Blood samples for determination of $A C L$ and $A C W$ were collected before and $5 \mathrm{~min}, 60 \mathrm{~min}$ and $6 \mathrm{~h}$ after induction to anaesthesia. In MMVD/PS dogs, ACL was significantly higher at all sampling times when compared to PS dogs. Compared to basal values, only anaesthesia maintained with propofol significantly increased $A C L$ at 60 min in dogs with MMVD. In MMVD/P dogs, ACW increased after induction to anaesthesia and remained elevated up to $6 \mathrm{~h}$ after anaesthesia. Compared to basal values, anaesthesia maintained with sevoflurane significantly increased ACW only at 60 min in both dogs with and without MMVD. The only difference between propofol and propofol/sevoflurane anaesthesia in dogs with MMVD was significantly higher ACW at 60 min after induction to anaesthesia in the propofol group.
\end{abstract}

Conclusions: Regarding antioxidant capacity, propofol could be a better choice than sevoflurane for anaesthesia of dogs with early-stage MMVD, although further studies are necessary to clarify the advantage of this antioxidant capacity.

Keywords: Dog, Cardiac disease, Antioxidant capacity, Anaesthesia

\footnotetext{
* Correspondence: alenka.seliskar@vf.uni-lj.si

${ }^{\dagger}$ Katerina Tomsič and Alenka Nemec Svete contributed equally to this work.

${ }^{1}$ Small Animal Clinic, Veterinary Faculty, University of Ljubljana, Gerbičeva 60, 1000 Ljubljana, Slovenia

Full list of author information is available at the end of the article
} 


\section{Background}

The heart is constantly subjected to ROS formation due to the high rate of aerobic metabolism [1]. Antioxidants located in both the hydrophilic and lipophilic compartments of plasma are actively involved in a defence system against ROS [2]. In dogs with MMVD, a disruption in the balance between ROS formation and antioxidant mechanisms leads to oxidative stress which may contribute to the pathogenesis and progression of the disease [3-6]. During anaesthesia, the metabolism of anaesthetics and changes in tissue oxygenation increase formation of ROS $[7,8]$ and induce cardiac injury [7, 9] by aggravating oxidative stress [1].

Propofol and sevoflurane are commonly used anaesthetics in dogs. Propofol has a structural feature, the phenolic hydroxyl group, like vitamin $\mathrm{E}$, and is believed to act as a ROS scavenger [10,11]. Sevoflurane, on the other hand, may promote ROS formation through its metabolism [12] and by influencing mitochondrial function [13].

In order to determine the most appropriate anaesthesia protocol for the dogs with early-stage MMVD, we determined the antioxidant capacity of lipid- and watersoluble antioxidants in dogs with MMVD which were anaesthetised with propofol alone, or in combination with sevoflurane, for a dental procedure. We hypothesised that total intravenous anaesthesia with propofol increases ACL and ACW compared with anaesthesia induced with propofol and maintained with sevoflurane in dogs with MMVD.

\section{Results}

Eighteen dogs diagnosed with MMVD ACVIM class B1 and B2, 7 females (3 neutered, 4 intact) and 11 males (3 neutered, 8 intact), weighing $17.52 \pm 8.86 \mathrm{~kg}$ and aged $8.65 \pm 3.05$ years were included in the study. The control group (PS) consisted of 12 dogs with no cardiac disease, 6 females (all neutered) and 6 males (2 neutered, 4 intact), weighing $23.11 \pm 9.08 \mathrm{~kg}$ and aged $5.88 \pm 2.45$ years.
Some samples for determination of ACW were excluded from the study due to technical problems with latent fibrin formation and the results are reported only for 7 dogs in the MMVD/PS group, 10 dogs in the PS group and 8 dogs in the MMVD/P group.

There were no differences between the groups in periodontal/dental disease status $(p=0.443)$, antibiotic administration $(p=0.443)$, use of regional nerve blocks $(p=0.054)$, age of dogs $(p=0.054)$ and duration of anaesthesia $(p=0.254)$. Ketamine was administered significantly more frequently $(p=0.017)$ in dogs with MMVD compared to dogs with no cardiac disease.

ACL values were significantly lower in the PS group compared to those in the MMVD/PS group at all sampling times. Compared to basal values, ACL increased significantly $60 \mathrm{~min}$ after induction to anaesthesia in the MMVD/P group (Table 1). The ACL value of the propofol formulation used in the study was $3.406 \mu \mathrm{mol}$ of trolox equivalents per millilitre of propofol solution containing $56.1 \mu \mathrm{mol}$ of propofol in equal quantity.

Compared to basal values, ACW increased significantly 5 min after induction to anaesthesia and remained elevated until $6 \mathrm{~h}$ after induction only in the MMVD/P group. At $60 \mathrm{~min}$ after induction to anaesthesia, ACW was also significantly increased in the MMVD/PS and PS groups. However, ACW was significantly higher in the MMVD/P group compared to the MMVD/PS group only at $60 \mathrm{~min}$ after induction to anaesthesia (Table 1).

\section{Discussion}

In an attempt to elucidate the effect of anaesthetics on oxidative status in dogs with MMVD, this study was set to evaluate the influence of propofol or sevoflurane on plasma water- and lipid-soluble antioxidants in dogs with early-stage MMVD undergoing periodontal treatment under general anaesthesia. The MMVD is the most common acquired cardiovascular disease in dogs with a long preclinical period [14]. Frequently, dogs with an early stage MMVD are diagnosed during a preanaesthetic evaluation, performed for procedures that require

Table 1 Values of antioxidant capacity of lipid- (ACL) and water-soluble (ACW) antioxidants in dogs diagnosed with myxomatous mitral valve degeneration and anaesthetised with propofol and sevoflurane (MMVD/PS) or propofol (MMVD/P) and in control dogs anaesthetised with propofol and sevoflurane (PS)

\begin{tabular}{llllll}
\hline Variable & Group & Basal & $5 \mathrm{~min}$ & $60 \mathrm{~min}$ & $6 \mathrm{~h}$ \\
\hline ACL $[\mathrm{nmol} / \mathrm{L}]$ & MMVD/PS & $133.9 \pm 31.3$ & $130.9 \pm 29.9$ & $134.3 \pm 31.9$ & $131.8 \pm 26.2$ \\
& PS & $87.6 \pm 12.2^{\mathrm{a}}$ & $86.6 \pm 13.7^{\mathrm{a}}$ & $93.0 \pm 18.6^{\mathrm{a}}$ & $84.9 \pm 22.2^{\mathrm{a}}$ \\
& MMVD/P & $116.4 \pm 22.9$ & $118.2 \pm 19.9$ & $134.0 \pm 23.3^{*}$ & $119.1 \pm 24.6$ \\
$\mathrm{ACW}[\mathrm{nmol} / \mathrm{L}]$ & MMVD/PS & $35.1 \pm 8.9$ & $47.1 \pm 15.6$ & $52.8 \pm 17.2^{*}$ & $42.1 \pm 15.5$ \\
& PS & $29.6 \pm 9.6$ & $37.4 \pm 4.8$ & $44.3 \pm 8.2^{*}$ & $35.44 \pm 8.0$ \\
& MMVD/P & $26.5 \pm 9.6$ & $42.5 \pm 14.2^{*}$ & $79.2 \pm 22.0^{* *^{\mathrm{a}}}$ & $55.8 \pm 30.6^{*}$ \\
\hline
\end{tabular}

Data are presented as (mean \pm SD). Abbreviations: basal, basal values; 5 min, 5 min after induction to anaesthesia; 60 min, 60 min after induction to anaesthesia; 6 $\mathrm{h}, 6 \mathrm{~h}$ after induction to anaesthesia. ${ }^{*} p<0.05$ compared to basal values; ${ }^{a} p<0.05$ compared to MMVD/PS 
general anaesthesia, such as a periodontal treatment. A statistically significant association has been found between periodontal disease and cardiac disease [15].

Metabolism of anaesthetics and changes in tissue oxygenation during anaesthesia promote increased formation of ROS, which affects antioxidant defence system [7, 16, 17]. Propofol and sevoflurane are commonly used anaesthetics in dogs; however, there are only few reports on the effects of these anaesthetics on oxidative stress parameters, including total antioxidant capacity in dogs [18-20] and people [21-23]. So far, ACL and ACW have not been evaluated in dogs during propofol or sevoflurane anaesthesia.

The plasma antioxidant capacity of lipid-soluble antioxidants covers exogenous and endogenous lipophilic antioxidants, including vitamin $\mathrm{E}$, coenzyme $\mathrm{Q}_{10}$, and carotenoids. In the plasma of healthy people, vitamin $\mathrm{E}$ represents up to $75 \%$ of ACL $[24,25]$. A decrease in vitamin $\mathrm{E}$ (total tocopherol) during anaesthesia was demonstrated in dogs with MMVD [20], healthy dogs [26] and people [27, 28], as well as a decrease in alpha tocopherol in people $[29,30]$. Significantly higher plasma ACL was determined in the MMVD/ PS and MMVD/P groups at all sampling times when compared with the PS group in this study. This might mostly be due to increased mobilisation of vitamin $E$ and other lipid soluble antioxidants in response to enhanced ROS production in dogs with cardiac disease [31, 32]. However, we cannot prove that because the individual antioxidants were not measured in our study.

The significant increase of ACL 60 min after induction in the MMVD/P group might be due to the antioxidant properties of propofol. The antioxidant properties of propofol have already been revealed [10, 33-35]. To assess the possible contribution of propofol to ACL, we measured ACL in a sample of the same propofol formulation as we used in the study and established that the propofol formulation has an extremely high ACL, which confirms its antioxidant properties. This is in accordance with studies of antioxidant capacity in human patients during propofol anaesthesia [21, 29, 36]. The lipid soluble component of blood antioxidant activity was evaluated in healthy women, and although propofol had only a small influence on total antioxidant capacity, there was an increase in the antioxidant protection of lipid membranes [36]. Furthermore, propofol accumulates in lipid membranes; thus, plasma may not be the best compartment to evaluate the antioxidant effect of propofol [21].

The antioxidant capacity of water-soluble antioxidants includes hydrophilic antioxidants such as vitamin $\mathrm{C}$, uric acid, glutathione, proteins and low molecular antioxidants $[2,25,37,38]$. Uric and ascorbic acids are major contributors to the ACW of human plasma, and they are included in the measurement of $\mathrm{ACW}$ by using the photochemiluminescence (PCL) method [25, 37-39]. The same method was used in our study. Popov and
Lewin $[25,37]$ reported that ACW determined with the PCL method is age-dependent and animals speciesspecific. Furthermore, stressors such as exercise, inflammation, or noise resulted in rapid increase of ACW values and ascorbic acid in men [25]. No differences in basal values of ACW were observed between the MMVD/PS and PS groups. These results could be due to homeostasis of water-soluble antioxidant mechanisms was not disrupted in dogs with early-stage MMVD or activation of compensatory mechanisms as enzymatic antioxidant system or increased synthesis of vitamin C. The only difference between the two anaesthesia protocols in dogs with MMVD was at 60 min after induction to anaesthesia, when significantly higher ACW was determined in dogs anaesthetised with propofol. Significantly elevated ACW $60 \mathrm{~min}$ after induction to anaesthesia in all groups, and at all sampling times after induction to anaesthesia in the MMVD/P group, may be attributed to the free radical scavenging activity of propofol [10, 34]. Similar results were reported for healthy people anaesthetised with propofol for elective surgery [29]. ACW constitutes mainly of antioxidative capacity of uric acid and antioxidative capacity of ascorbic acid $[25,38]$. Uric acid is proposed as a potent free-radical scavenger, the serum concentration of which rises in the settings of acute and chronic oxidative stress [40, 41]. Though less likely, another explanation for a significant increase in ACW in our dogs could be attributed to the increased concentrations of ascorbic acid and/or uric acid as a result of increased oxidative stress during anaesthesia. However, we cannot prove this because the concentration of uric acid or ascorbic acid was not measured in our dogs.

The limitation of this study is that the dogs were client-owned, and thus the environmental and nutritional backgrounds of the dogs differed, which may have influenced the antioxidant parameters [42]. In fact, the antioxidant status may have been affected by different levels of environmental pollution (including, for example, smoking in the household), and although the owners stated that the dogs were not given any vitamins or antioxidants and were mostly fed commercial food, the composition of their food may have differed in antioxidant and vitamin content. Other limitations of the study are the low number of dogs included in the study and the unequal distribution of the number of dogs per group. Because of the low number of dogs per group, post-hoc power analysis was conducted. Statistically nonsignificant results may be the result of insufficient power [43, 44]. Statistical analyses indicated enough power for ACL measurements (power coefficient of $0.843 ; n=30$ ), while a power coefficient for ACW measurements $(0.756 ; n=25)$ almost reached the recommended value of 0.80 [43]. 


\section{Conclusions}

Compared to basal values, ACW was significantly increased $60 \mathrm{~min}$ after induction to anaesthesia in both dogs with and without MMVD regardless of the anaesthesia protocol. In dogs with MMVD, ACW was significantly higher in the propofol group. In dogs with MMVD, only anaesthesia with propofol significantly increased ACL. These results suggest that regarding antioxidant capacity, propofol could be a better choice than sevoflurane for anaesthesia, although further studies are necessary to clarify the advantage of this antioxidant capacity in dogs with non-severe MMVD.

\section{Methods}

\section{Animals}

This prospective clinical study was evaluated and approved by the National Ethics Committee and written informed client consent was obtained before the dogs were included in the study. All procedures complied with the relevant Slovenian (Animal Protection Act UL RS, 43/2007) and European regulations. Client-owned dogs receiving no medication 1 month prior to anaesthesia were recruited from the University of Ljubljana Veterinary Faculty subject pool. Their health status was evaluated based on history, physical examination and blood tests including complete blood count with white cell differential count and serum biochemical analyses (glucose, urea, creatinine, sodium, potassium, chloride, calcium, total protein, albumin, alanine aminotransferase, alkaline phosphatase, serum total cholesterol and triglycerides). Cardiovascular disease was confirmed by history, clinical examination, standard electrocardiogram and echocardiography using two-dimensional, M-mode, and colour and spectral Doppler modes (VIVID E9, General Electric Healthcare, Milwaukee, Wisconsin, USA).

All eligible dogs that were presented between June 2016 and January 2017 were included. The final sample size was $n=30$. The dogs were diagnosed with periodontal disease and scheduled for a dental procedure under general anaesthesia. Eighteen dogs were diagnosed with MMVD class B1 or B2 according to the American College of Veterinary Internal Medicine (ACVIM) classification [45]. They were randomly assigned (by tossing a coin) to anaesthesia with propofol (MMVD/P, $n=10)$ or sevoflurane (MMVD/PS, $n=8$ ). The control group consisted of 12 dogs with no evidence of MMVD.

\section{Study protocol}

Dogs were premedicated with morphine $0.3 \mathrm{mg} / \mathrm{kg}$ administered subcutaneously (SC) 20 min before induction to anaesthesia. An intravenous catheter was placed into the cephalic vein, and after $5 \mathrm{~min}$ of preoxygenation (flow by, $2 \mathrm{~L} / \mathrm{min}$ ), anaesthesia was induced with propofol (3-6 mg/kg) intravenously (IV) and titrated to effect.
The dogs were intubated endotracheally within 30 to 60 $\mathrm{s}$, connected to a circle breathing system and allowed to breathe oxygen spontaneously. In dogs with MMVD, anaesthesia was maintained with propofol at $0.3-0.6 \mathrm{mg} /$ $\mathrm{kg} / \mathrm{min}$ IV (MMVD/P group) or with sevoflurane at an end-tidal sevoflurane concentration of 2 to 3\% (MMVD/ PS group). Dogs with no evidence of MMVD (PS group) were anaesthetised the same way as the MMVD/PS group. Hartmann's solution was infused at $5 \mathrm{~mL} / \mathrm{kg} / \mathrm{h} \mathrm{IV}$ during anaesthesia. Perioperative analgesia was supported with oral regional nerve blocks with levobupivacaine 1 to $2 \mathrm{mg} / \mathrm{kg}$ and/or IV boluses of ketamine 0.5 $\mathrm{mg} / \mathrm{kg}$. Ketamin was administered when the heart rate increased by $20 \%$ or if signs of nociceptive responses occurred during anaesthesia. Perioperative antibiotic management was carried out with cefazolin $20 \mathrm{mg} / \mathrm{kg}$ IV if clinically indicated.

The dogs were placed in dorsal recumbency and warmed with bags filled with warm water. End-tidal sevoflurane concentration, end-tidal $\mathrm{CO}_{2}$ tension, respiratory rate, ECG, blood pressure (non-invasively) and rectal temperature were monitored during anaesthesia.

Dogs with the majority of teeth present were included in the study, and a detailed oral examination (probing, charting and full-mouth dental radiographs) was performed prior to periodontal treatment. Dogs were divided into two groups based on their oral/dental disease for statistical analysis: $\leq 25 \%$ of the teeth affected with periodontitis and/or dental fractures and $>25 \%$ of the teeth affected with periodontitis and/or dental fractures.

During recovery, the dogs were administered Hartmann's solution at $2 \mathrm{~mL} / \mathrm{kg} / \mathrm{h}$ IV and morphine $0.3 \mathrm{mg} /$ $\mathrm{kg} \mathrm{SC}$ every $3 \mathrm{~h}$, depending on the invasiveness of the procedure. Carprofen $4 \mathrm{mg} / \mathrm{kg}$ IV was administered after the last blood sampling. All dogs were released to home care the same day, with analgesics and antibiotics prescribed as clinically indicated. They were prescribed carprofen $2 \mathrm{mg} / \mathrm{kg}$ orally twice daily for 4 to 6 days. In case of severe acute pain, a transdermal fentanyl patch (3 to $4 \mu \mathrm{g} / \mathrm{kg} / \mathrm{h}$ ) was placed on the skin of the lateral thoracic region at the end of anaesthesia.

\section{Blood sample collection, processing, and analyses}

Venous blood samples were collected from the jugular vein before premedication (basal values) and $5 \mathrm{~min}$ (immediate post induction period), $60 \mathrm{~min}$ (intraoperative period) and $6 \mathrm{~h}$ after induction to anaesthesia (postoperative period).

Blood samples for determination of ACL and ACW concentrations (total volume $2 \mathrm{~mL}$ ) were collected in lithium heparin containing tubes (Vacuette, Greiner BioOne, Kremsmunster, Austria). Samples were immediately centrifuged at $1500 \times \mathrm{g}$ for $15 \mathrm{~min}$ at $4{ }^{\circ} \mathrm{C}$ and plasma immediately frozen at $-80^{\circ} \mathrm{C}$ until analysis. 
Determination of antioxidant capacity of water- and lipidsoluble antioxidants

The method is based on the chemiluminometric detection of photochemically generated superoxide anion radicals from a photosensitizer (luminol), which are partially eliminated from the sample by reaction with antioxidants present in the sample. The remaining radicals react with luminol to produce luminescence, which is measured with a PHOTOCHEM analyser (Analytik Jena, Jena, Germany) using supplied reagent kits. After defrosting and vortexing, $100 \mu \mathrm{L}$ of plasma sample and $100 \mu \mathrm{L}$ of methanol were pipetted in a $1.5 \mathrm{~mL}$ plastic container with a cap. Samples were then vortexed for $10 \mathrm{~min}$ and centrifuged at 25,000 $\mathrm{rpm}$ for $10 \mathrm{~min}$ at $4{ }^{\circ} \mathrm{C}$. Sample preparation steps were performed in a dark place. Until analysis (usually within 30 $\mathrm{min}$ ), prepared samples were held in the dark at a temperature below $4{ }^{\circ} \mathrm{C}$. Plasma ACL and ACW were measured in accordance with the manufacturer instructions, using reagent kits (Analytik Jena, Jena, Germany) with the PHOTOCHEM analyser. The results of the ACW measurements are expressed in nmol equivalents of ascorbic acid in $\mathrm{mL}$ of the sample, and the results of the ACL measurements in nmol equivalents of trolox (6-hydroxy-2,5,7,8-tetramethylchroman-2-carboxylic acid) in $\mathrm{mL}$ of the sample.

To assess whether the propofol formulation used in the study has antioxidative properties, we also measured ACL in a sample of propofol formulation, using the same method as described above.

\section{Statistical analysis}

An a priori sample size calculation was not performed as no comparable data from the literature regarding ACW and ACL were available to enable calculation. Post-hoc sample size calculation indicated that with an effect size of 0.5 and significance level $p=0.05,30$ dogs would be enough to achieve more than $80 \%$ power of the study. Statistical analysis was supported by the R statistical software program (version 3.2.2) with the nlme package [46]. The Kruskal-Wallis test was used to evaluate the differences in the baseline characteristics of the dogs, age and duration of anaesthesia. Fisher's exact test was used to evaluate the differences between the MMVD/PS, MMVD/ $\mathrm{P}$ and PS groups with respect to the baseline characteristics of periodontal/dental disease status and the use of antibiotics, ketamine and nerve blocks. A linear mixed-effect analysis was used to examine the treatment effect and trends over time within groups (MMVD/PS, MMVD/P and PS) and between groups (the MMVD/PS group was compared to the MMVD/P and PS groups). The models included a random intercept for each dog and three fixed effects: time, group and interaction term. In case of significant fixed effects, multiple comparisons were performed using Holm-Bonferroni correction. A value of $p<0.05$ was considered significant.

\section{Abbreviations}

ACL: Antioxidant capacity of lipid-soluble antioxidants; ACW: Antioxidant capacity of water-soluble antioxidants; IV: intravenously; MMVD: myxomatous mitral valve degeneration; MMVD/P: dogs with MMVD and anaesthetised with propofol; MMVD/PS: dogs with MMVD and anaesthetised with propofol and sevoflurane; PS: dogs with no evidence of MMVD and anaesthetised with propofol and sevoflurane; ROS: reactive oxygen species;

SC: subcutaneously

\section{Acknowledgments}

The authors thank the staff of the Small Animal Clinic, Veterinary Faculty, University of Ljubljana, Slovenia for technical support. We also thank Professor lgor Popov, one of the two patent holders of the PCL method for measurement of ACW and ACL and author of several references used in this manuscript for consultancy.

\section{Authors' contributions}

AS and ANS designed the experiment. KT, AN, ADP and AS performed clinical experiments. TP, VR, TV and ANS performed laboratory analyses and interpreted the results. KT, AN, ANS and AS drafted the manuscript. AS and ANS reviewed the manuscript. All authors read and approved the final manuscript.

\section{Funding}

This study was supported by the Slovenian Research Agency (P4-0053, P40097, P1-0189). The funders were not involved in the study design; collection, analysis and interpretation of the data; or in writing the manuscript.

\section{Availability of data and materials}

The datasets used and/or analysed during the current study are available from the corresponding author on reasonable request.

\section{Ethics approval and consent to participate}

All procedures complied with the relevant Slovenian (Animal Protection Act UL RS, 43/2007) and European (Directive 2010/63/EU) guidelines. All procedures involving the use of animals were approved by the National Ethics Committee (license No U34401-38/2013/2, approval date 30.7.2013), and written informed client consent was obtained before the dogs were included in the study.

\section{Consent for publication}

Not applicable.

\section{Competing interests}

The authors declare that they have no competing interest.

\section{Author details}

${ }^{1}$ Small Animal Clinic, Veterinary Faculty, University of Ljubljana, Gerbičeva 60, 1000 Ljubljana, Slovenia. ${ }^{2}$ Department of Animal Science, Biotechnical Faculty, University of Ljubljana, Groblje 3, 1230 Domžale, Slovenia. ${ }^{3}$ Faculty of Pharmacy, University of Ljubljana, Aškerčeva 7, 1000 Ljubljana, Slovenia.

Received: 13 March 2020 Accepted: 18 August 2020

Published online: 24 August 2020

\section{References}

1. Martín-Fernández B, Gredilla R. Mitochondria and oxidative stress in heart aging. Age. 2016;38:225-38 https://doi.org/10.1007/s11357-016-9933-y.

2. Yeum K-J, Russell RM, Krinsky NI, Aldini G. Biomarkers of antioxidant capacity in the hydrophilic and lipophilic compartments of human plasma. Arch Biochem Biophys. 2004;430:97-103 https://doi.org/10.1016/j.abb.2004.03.006.

3. Freeman LM, Rush JE, Milbury PE, Blumberg JB. Antioxidant status and biomarkers of oxidative stress in dogs with congestive heart failure. J Vet Intern Med. 2005;19:537-41 https://doi.org/10.1892/0891-6640(2005)19[537: asaboo]2.0.co;2.

4. Freeman LM, Rush JE, Markwell PJ. Effects of dietary modification in dogs with early chronic valvular disease. J Vet Intern Med. 2006;20:1116-26 https://doi.org/10.1892/0891-6640(2006)20[1116:eodmid]2.0.co;2.

5. Reimann MJ, Häggström J, Mortensen A, Lykkesfeldt J, Møller JE, Falk T, et al. Biopterin status in dogs with myxomatous mitral valve disease is 
associated with disease severity and cardiovascular risk factors. J Vet Intern Med. 2014;28:1520-6 https://dx.doi.org/10.1111\%2Fjvim.12425.

6. Li Q, Heaney A, Langenfeld-McCoy N, Vester Boler B, Laflamme DP. Dietary intervention reduces left atrial enlargement in dogs with early preclinical myxomatous mitral valve disease: a blinded randomized controlled study in 36 dogs. BMC Vet Res. 2019;15:425 https://doi.org/ 10.1186/s12917-019-2169-1.

7. Kevin LG, Novalija E, Riess ML, Camara AK, Rhodes SS, Stowe DF. Sevoflurane exposure generates superoxide but leads to decreased superoxide during ischemia and reperfusion in isolated hearts. Anesth Analg. 2003;96:949-55 https://doi.org/10.1213/01.ane.0000052515.25465.35.

8. Sleeper MM, Rosato BP, Bansal S, Avadhani NG. Mitochondrial dysfunction in myocardium obtained from clinically normal dogs, clinically normal anesthetized dogs, and dogs with dilated cardiomyopathy. Am J Vet Res. 2012;73:1759-64 https://doi.org/10.2460/ajvr.73.11.1759.

9. Kevin LG, Novalija E, Stowe DF. Reactive oxygen species as mediators of cardiac injury and protection: the relevance to anesthesia practice. Anesth Analg. 2005;101:1275-87 https://doi.org/10.1213/01.ane.0000180999.81013.d0.

10. Volti LG, Murabito P, Attaguile G, Rodella LF, Astuto M, Di Giacomo C, et al. Antioxidant properties of propofol: when oxidative stress sleeps with patients. EXCLI J. 2006:5:25-32.

11. Murphy PG, Myers DS, Davies MJ, Webster NR, Jones JG. The antioxidant potential of propofol (2,6-diisopropyl-phenol). Br J Anaesthes. 1992;68:613-8 https://doi.org/10.1093/bja/68.6.613.

12. Kharasch ED. Biotransformation of sevoflurane. Anesth Analg. 1995;81:27-3 https://doi.org/10.1097/00000539-199512001-000058.

13. Sedlic F, Pravdic D, Ljubkovic M, Marinovic J, Stadnicka A, Bosnjak ZJ. Differences in production of reactive oxygen species and mitochondrial uncoupling as events in the preconditioning signaling cascade between desflurane and sevoflurane. Anesth Analg. 2009;109:405-11 https://dx.doi. org/10.1213\%2Fane.0b013e3181a93ad9.

14. Borgarelli M, Haggstrom J. Canine degenerative myxomatous mitral valve disease: natural history, clinical presentation and therapy. Vet Clin North Am Small Anim Pract. 2010;40:651-63 https://doi.org/10.1016/j. cvsm.2010.03.008.

15. Pereira Dos Santos JD. Cunha E, Nunes T, Tavares L, Oliveira M. relation between periodontal disease and systemic diseases in dogs. Res Vet Sci. 2019;125:136-40 https://doi.org/10.1016/j.rvsc.2019.06.007.

16. El-Bassiouni EA, Abo-Ollo MM, Helmy MH, Ismail S, Ramadan MI. Changes in the defense against free radicals in the liver and plasma of the dog during hypoxia and/or halothane anaesthesia. Toxicology. 1998;128:25-34 https:// doi.org/10.1016/s0300-483x(98)00045-6.

17. Kovacic P, Somanathan R. Mechanism of anesthetic toxicity: metabolism, reactive oxygen species, oxidative stress, and electron transfer. ISRN Anesthesiol. 2011:1-10 https://doi.org/10.5402/2011/402906.

18. Lee JY. Oxidative stress due to anesthesia and surgical trauma and comparison of the effects of propofol and thiopental in dogs. J Vet Med Sci. 2012;74:663-5 https://doi.org/10.1292/jvms.11-0221.

19. Lee JY, Kim MC. Effect of propofol on oxidative stress status in erythrocytes from dogs under general anaesthesia. Acta Vet Scand. 2012;54:76 https://doi.org/10.1186/1751-0147-54-76.

20. Tomsič K, Nemec Svete A, Nemec A, Domanjko Petrič A, Vovk T, Seliškar A. Influence of sevoflurane or propofol anaesthesia on oxidative stress parameters in dogs with early-stage myxomatous mitral valve degeneration. A preliminary study. Acta Vet-Beograd. 2018;68:32-42 https://doi.org/10. 2478/acve-2018-0003.

21. Hans P, Deby-Dupont G, Deby C, Pieron F, Verbesselt R, Franssen C, Lamy $M$. Increase in antioxidant capacity of plasma during propofol anesthesia. J Neurosurg Anesthesiol. 1997;9:234-6 https://doi.org/10.1097/00008506199707000-00006.

22. Cinnella G, Vendemiale G, Dambrosio M, Serviddio G, Pugliese PL, Aspromonte G, et al. Effect of Propofol, Sevoflurane and Desflurane on systemic redox balance. Int J Immunopathol Pharmacol. 2007;20:585-93 https://doi.org/10.1177/039463200702000316.

23. Erbas M, Demiraran Y, Yildirim HA, Sezen G, Iskender A, Karagoz I, et al. Comparison of effects on the oxidant/antioxidant system of sevoflurane, desflurane and propofol infusion during general anesthesia. Rev Bras Anestesiol. 2015;65:68-72 https://doi.org/10.1016/j.bjane.2014.05.004.

24. Popov IN, Lewin G. Photochemiluminescent detection of antiradical activity; IV: testing of lipid-soluble antioxidants. J Biochem Biophys Methods. 1996; 31:1-8 https://doi.org/10.1016/0165-022X(95)00021-I.
25. Popov I, Lewin G. Antioxidative homeostasis: characterization by means of chemiluminescent technique. Methods Enzymol. 1999;300:437-56 https://doi.org/10.1016/s0076-6879(99)00149-4.

26. Naziroǧlu M, Günay C. The levels of some antioxidant vitamins, glutathione peroxidase and lipoperoxidase during the anaesthesia of dogs. Cell Biochem Funct. 1999;17:207-12 https://doi.org/10.1002/(SICI)10990844(199909)17:3\%3C207::AID-CBF830\%3E3.0.CO;2-3.

27. Ceylan BG, Yilmaz F, Eroglu F, Yavuz L, Gulmen S, Vural H. Oxidant and antioxidant activities of different anesthetic techniques. Propofol versus desflurane. Saudi Med J. 2009;30:371-6.

28. Eroglu F, Yavuz L, Ceylan BG, Yilmaz F, Eroglu E, Delibas N, et al. New volatile anesthetic, desflurane, reduces vitamin $E$ level in blood of operative patients via oxidative stress. Cell Biochem Funct. 2010;28:211-6 https://doi. org/10.1002/cbf.1641.

29. Braz MG, Braz LG, Freire CMM, Lucio LMC, Braz JRC, Tang G, et al. Isoflurane and propofol contribute to increasing the antioxidant status of patients during minor elective surgery: a randomized clinical study. Medicine (Baltimore). 2015;94(31):e1266 https://doi.org/10.1097/md. 0000000000001266.

30. Cavalca V, Colli S, Veglia F, Eligini S, Zingaro L, Squellerio I, et al. Anesthetic propofol enhances plasma gamma-tocopherol levels in patients undergoing cardiac surgery. Anesthesiology. 2008;108:988-97 https://doi.org/10.1097/ ALN.0b013e318173efb4

31. Liebler DC. The role of metabolism in the antioxidant function of vitamin $E$. Crit Rev Toxicol. 1993;23:147-69 https://doi.org/10.3109/ 10408449309117115

32. Palace VP, Hill MF, Farahmand F, Singal PK. Mobilization of antioxidant vitamin pools and hemodynamic function after myocardial infarction. Circulation. 1999;99:121-6 https://doi.org/10.1161/01.CIR.99.1.121.

33. Boisset S, Steghens J-P, Favetta P, Terreux R, Guitton J. Relative antioxidant capacities of propofol and its main metabolites. Arch Toxicol. 2004;78:63542 https://doi.org/10.1007/s00204-004-0585-9.

34. Rigobello MP, Stevanato R, Momo F, Fabris S, Scutari G, Boscolo R, et al. Evaluation of the antioxidant properties of propofol and its nitrosoderivative. Comparison with homologue substituted phenols. Free Radic Res. 2004;38:315-21 https://doi.org/10.1080/03079450310001652031.

35. Vasileiou I, Xanthos T, Koudouna E, Perrea D, Klonaris C, Katsargyris A, et al. Propofol: a review of its non-anaesthetic effects. Eur J Pharmacol. 2009;605: 1-8 https://doi.org/10.1016/j.ejphar.2009.01.007.

36. Stratford N, Murphy P. Antioxidant activity of propofol in blood from anaesthetized patients. Eur J Anaesthesiol. 1998;15:158-60 https://doi.org/ 10.1111/j.0265-0215.1998.00261.x

37. Popov IN, Lewin G. Photochemiluminescent detection of antiradical activity: II. Testing of nonenzymic water-soluble antioxidants. Free Radic Biol Med. 1994;17:267-71 https://doi.org/10.1016/0891-5849(94)90082-5.

38. Popov IN, Lewin G. Antioxidative system of the organism and thermoinitiated chemiluminescence method for quantitative evaluation of its state. Biophysics. 2013;58:669-75 https://doi.org/10.1134/S0006350913050138.

39. Popov I, Lewin G. Antioxidative homeostasis, its evaluation by means of chemiluminescent methods. In: Popov I, Lewin G, editors. Handbook of Chemiluminescent methods in oxidative stress assessment. Kerala: Transworld Research Network; 2008. p. 361-91.

40. Waring WS, Webb DJ, Maxwell SRJ. Systemic uric acid administration increases serum antioxidant capacity in healthy volunteers. J Cardiovasc Pharmacol. 2001;38:365-71 https://doi.org/10.1097/ 00005344-200109000-00005.

41. Glantzounis GK, Tsimoyiannis EC, Kappas AM, Galaris DA. Uric acid and oxidative stress. Curr Pharm Des. 2005;11:4145-51 https://doi.org/10.2174/ 138161205774913255

42. Ghiselli A, Serafini M, Natella F, Scaccini C. Total antioxidant capacity as a tool to assess redox status: critical view and experimental data. Free Radic Biol Med. 2000;29:1106-14 https://doi.org/10.1016/s08915849(00)00394-4.

43. Onwuegbuzie AJ, Leech NL. Post hoc power: a concept whose time has come. Understand Stat. 2004;3:201-30 https://doi.org/10.1207/ s15328031us0304_1.

44. Yuan KH, Maxwell S. On the post hoc power in testing mean differences. J Educ Behav Stat. 2005;30:141-67 https://doi.org/10.3102\%2 F10769986030002141.

45. Atkins $C$, Bonagura J, Ettinger S, Fox P, Gordon S, Haggstrom J, et al. Guidelines for the diagnosis and treatment of canine chronic valvular heart 
disease. J Vet Intern Med. 2009;23:1142-50 https://doi.org/10.1111/j.19391676.2009.0392.x.

46. Pinheiro J, Bates D, DebRoy S, Sarkar D, R Development Core Team, R. nIme: linear and nonlinear mixed effects models 2015. R Package version 3.1-142 R package, 1-3. Available from: https://cran.r-project.org/web/packages/ nlme/nlme.pdf.

\section{Publisher's Note}

Springer Nature remains neutral with regard to jurisdictional claims in published maps and institutional affiliations.

Ready to submit your research? Choose BMC and benefit from:

- fast, convenient online submission

- thorough peer review by experienced researchers in your field

- rapid publication on acceptance

- support for research data, including large and complex data types

- gold Open Access which fosters wider collaboration and increased citations

- maximum visibility for your research: over $100 \mathrm{M}$ website views per year

At $\mathrm{BMC}$, research is always in progress.

Learn more biomedcentral.com/submissions 